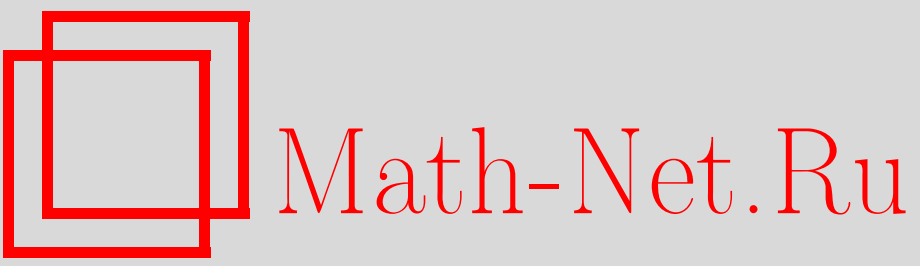

А. А. Тарасов, Максимальность некоторых коммутативных подалгебр в алгебрах Пуассона полупростых алгебр Ли, УМH, 2002, том 57, выпуск 5, 165-166

DOI: https://doi.org/10.4213/rm567

Использование Общероссийского математического портала Math-Net.Ru подразумевает, что вы прочитали и согласны с пользовательским соглашением

http://www.mathnet.ru/rus/agreement

Параметры загрузки:

IP : 3.80 .253 .173

26 апреля 2023 г., 14:00:20 


\title{
МАКСИМАЛЬНОСТЬ НЕКОТОРЫХ КОММУТАТИВНЫХ ПОДАЛГЕБР В АЛГЕБРАХ ПУАССОНА ПОЛУПРОСТЫХ АЛГЕБР ЛИ
}

\author{
A. A. TAPACOB
}

Пусть $\mathfrak{g}$ - полупростая комплексная алгебра Ли, $S(\mathfrak{g})$ - ее симметрическая алгебра, $P(\mathfrak{g})$ - ее алгебра Пуассона, т.е. алгебра $S(\mathfrak{g})$, снабженная скобкой Пуассона-Ли.

Элементы алгебры $P(\mathfrak{g})$ будем рассматривать как функции на $\mathfrak{g}^{*}$ или на $\mathfrak{g}$, отождествляя $\mathfrak{g}$ и $\mathfrak{g}^{*}$ при помощи инвариантного скалярного умножения $(\cdot, \cdot)$. Пусть $\left\{f_{i} \in P(\mathfrak{g}) \mid i=1, \ldots, r\right\}$ есть алгебраически независимая система порождающих центра алгебры $P(\mathfrak{g})$. Пусть $\mathfrak{h} \subset \mathfrak{g}-$ картановская подалгебра, $h \in \mathfrak{h}$ - регулярньй элемент. Тогда элементы $\partial_{h}^{k} f_{i}(i=1, \ldots, r$; $\left.k=0,1, \ldots, \operatorname{deg} f_{i}-1\right)$ алгебры $P(\mathfrak{g})$ попарно коммутируют и алгебраически независимы [1]. Порожденную ими коммутативную подалгебру обозначим через $F(h)$. Она имеет максимальную возможную степень трансцендентности среди коммутативных подалгебр алгебры $P(\mathfrak{g})$. Подалгебры вида $F(h)$ назьваются подалгебрами Мищенко-Фоменко.

Основным утверждением данной работы является следующая

Tеорема 1. $F(h)$ есть максимальная коммутативная подалгебра алгебры $P(\mathfrak{g})$.

Пусть $\boldsymbol{a}=a(t)$ - такой сходящийся (в окрестности нуля) степенной ряд с коэффицциентами из алгебры $\mathfrak{h}$, что элемент $a(t)$ регулярен и полупрост при всех достаточно малых $t \neq 0$.

Пусть $F(\boldsymbol{a})=\lim _{t \rightarrow 0} F(a(t))($ см. [2], [3]). В [3] доказано, что $F(\boldsymbol{a})$ есть свободная коммутативная подалгебра максимальной степени трансцендентности.

Теорема 2. $F(\boldsymbol{a})$ есть максимальная коммутативная подалгебра алгебры $P(\mathfrak{g})$.

Теорема 1 следует из теоремы 2. При доказательстве будет использоваться основное свойство, которое для подалгебр вида $F(h)$ было доказано в [1], а для предельных подалгебр - в [3].

Основноє свойство. Пусть $e$ - сумма всех корневых векторов, отвечающих простым корням относительно $\mathfrak{h}$. Тогда существует набор $\left\{f_{\gamma} \mid \gamma \in \Gamma\right\}$ однородных свободных порождающих подалгебры $F(\boldsymbol{a})$ такой, что векторы $\left\{d_{e} f_{\gamma} \mid \gamma \in \Gamma\right\}$ линейно независимы.

Автор глубоко признателен своему научному руководителю Э.Б. Винбергу за постановку задачи, а также за постоянное внимание к работе.

1. Лемма 1. Пусть $A$ - целостное кольцо, допускающее разложение вида $A=B \oplus I$, где $B$ - подкольцо, $I-$ идеал кольца $A$. Тогда $B$ алгебраически замкнуто в $A$.

ДокАЗАтЕльство. Пусть это не так, т.е. существует элемент $c \notin B$, алгебраический над $B$. Пусть в силу разложения $A=B \oplus I$ элемент $c$ представим в виде $c=b+u, b \in B, u \in I$, причем $u \neq 0$. Тогда элемент $u=c-b$ также алгебраичен над $B$. Рассмотрим для него минимальньй аннулирующий многочлен $b_{0}+b_{1} u+\cdots+b_{m} u^{m}=0, b_{0}, \ldots, b_{m} \in B$. Тогда $b_{0} \neq 0$ в силу минимальности многочлена, с другой стороны, $b_{1} u+\cdots+b_{m} u^{m}=u\left(b_{1}+\cdots+b_{m} u^{m-1}\right) \in I$, откуда $b_{0}=0$. Получаем противоречие.

2. Множество $\Delta$ всех корней алгебры Ли $\mathfrak{g}$ разлагается в объединение $\Delta=\bigcup_{i \neq 0} \Delta_{i}$, где $\Delta_{i}=-\left(\Delta_{-i}\right)$ при $i>0$ состоит из тех положителных корней, которые разлагаются в сумму ровно $i$ простых корней. Пусть $\mathfrak{g}_{i}=\left\langle e_{\alpha} \mid \alpha \in \Delta_{i}\right\rangle, \mathfrak{g}_{0}=\mathfrak{h}$. Положим $\mathfrak{g}^{+}=\sum_{i \geqslant 1} \mathfrak{g}_{i}, \mathfrak{b}=\mathfrak{g}^{+} \oplus \mathfrak{h}$ и $\mathfrak{q}=\mathfrak{b} \oplus \mathbb{C} e_{-}$, где $e_{-}=\sum_{\alpha \in \Delta_{-1}} e_{\alpha}$. Без ограничения общности будем считать, что $\left(e, e_{-}\right)=1$. Определим проекцию $Q: \mathfrak{g} \rightarrow \mathfrak{q}$ следующим образом: $\left.Q\right|_{\mathfrak{b}}=\operatorname{Id},\left.Q\right|_{\sum_{i \leqslant-2} \mathfrak{g}_{i}}=0, Q\left(e_{\alpha}\right)=$ $\frac{1}{\mathrm{rk} \mathfrak{g}} e_{-}$при $e_{\alpha} \in \mathfrak{g}-1$. Продолжим ее до гомоморфизма $Q: S(\mathfrak{g}) \rightarrow S(\mathfrak{q})$ симметрических алгебр. Ясно, что алгебра $S(\mathfrak{q})$ линейно порождена мономами вида

$$
\prod_{i=1}^{s_{1}} e_{\beta_{i}} \prod_{j=1}^{s_{2}} h_{\alpha_{j}} \cdot e_{-}^{s_{3}},
$$

Работа выполнена при частичной поддержке гранта CRDF RM1-2088. 
где $\beta_{i} \in \bigcup_{t \geqslant 1} \Delta_{t}, \alpha_{j} \in \Delta_{1}, e_{\beta_{i}}$ - корневой вектор, отвечающий корню $\beta_{i}$. Пусть $h_{0} \in \mathfrak{h}$ - такой элемент, что $\alpha\left(h_{0}\right)=1$ для любого корня $\alpha \in \Delta_{1}$. Пусть $S(\mathfrak{q})^{h_{0}}=\left\{x \in S(\mathfrak{q}) \mid\left\{h_{0}, x\right\}=0\right\}$. Отметим, что $Q$ перестановочно с действием $\operatorname{ad}\left(h_{0}\right)$.

3. ЛЕМма 2. $Q\left(P(\mathfrak{g})^{\mathfrak{h}}\right)=S(\mathfrak{q})^{h_{0}}$.

ДокАЗАТЕЛЬСтво. Пусть в мономе вида (1) $\beta_{i} \in \Delta_{p_{i}}, p_{i}>0$ при $i=1, \ldots, s_{1}$. Ясно, что этот моном лежит в $Q\left(P(\mathfrak{g})^{\mathfrak{h}}\right)$ тогда и только тогда, когда $s_{3}=\sum_{i=1}^{s_{1}} p_{i}$.

Отсюда следует, что $Q\left(P(\mathfrak{g})^{\mathfrak{h}}\right)$ свободно порождается элементами $e_{\beta} e_{-}^{s}, \beta \in \Delta_{s}, s>0$, и $h_{\alpha}, \alpha \in \Delta_{1}$. Очевидно, что эти же элементы свободно порождают $S(\mathfrak{q})^{h_{0}}$. Лемма доказана.

4. Пусть $\pi: S(\mathfrak{q})^{h_{0}} \rightarrow S(\mathfrak{b})$ - изоморфизм, которьй определен на порождающих алгебры $S(\mathfrak{q})^{h_{0}}$ следующим образом: $\pi\left(e_{\beta} e_{-}^{s}\right)=e_{\beta}, \beta \in \Delta_{s}, s>0 ; \pi\left(h_{\alpha}\right)=h_{\alpha}, \alpha \in \Delta_{1}$.

Лемма 3. Пусть $y \in S^{p}(\mathfrak{q})^{h_{0}}, p \in \mathbb{N}$. Тогда $\pi(y) \equiv d_{e} y \bmod S\left(\sum_{j=0}^{p-2} \mathfrak{g}_{j}\right)$, причем $d_{e} y \in \mathfrak{g}_{p-1}$.

ДОКАЗАТЕЛЬСТвО. При $p=1$ утверждение очевидно. При $p \geqslant 2$ пусть mon - произвольный моном из разложения $y$ по базисным мономам вида (1). Пусть корневой вектор $e_{\beta}, \beta \in \Delta_{t}$, входит в разложение (1) монома mon. Тогда в силу соотношения $s_{3}=\sum_{i=1}^{s_{1}} p_{i}$ и единственности разложения $\beta$ по элементам из $\Delta_{1}$ ясно, что deg mon $\geqslant t+1$, поскольку mon содержит как минимум еще $t$ компонент вида $e_{-}$. Отсюда получаем, что в силу условия $\operatorname{deg} y=p$, во-первых, в разложении (1) для mon нет ни одного корневого вектора из $\sum_{i \geqslant p} \mathfrak{g}_{i}$. Во-вторых, если в разложении (1) для mon есть корневой вектор из $\mathfrak{g}_{p-1}$, то кроме него в разложении нет больше ни одного корневого вектора из $\mathfrak{g}^{+}$, поскольку все остальные компоненты монома mon в силу соотношения $s_{3}=\sum_{i=1}^{s_{1}} p_{i}$ определены однозначно и имеют вид $e_{-}$. Отсюда $\pi(y)=A+B$, где $A \in \mathfrak{g}_{p-1}, B \in S\left(\sum_{i=0}^{p-2} \mathfrak{g}_{i}\right)$. Покажем, что $A=d_{e} y$. Последнее достаточно показать для $y=e_{\beta} e_{-}^{p-1}, \beta \in \Delta_{p-1}$, так как $d_{e} y \neq 0$ толшко для таких мономов. Осталось заметить, что $\pi\left(e_{\beta} e_{-}^{p-1}\right)=e_{\beta}=d_{e}\left(e_{\beta} e_{-}^{p-1}\right)$.

5. ЛЕмма 4. Ограничение $\left.Q\right|_{F(\boldsymbol{a})}: F(\boldsymbol{a}) \rightarrow S(\mathfrak{q})^{h_{0}}$ есть изоморфизм алгебр.

ДокАЗАТЕЛЬСТво. В силу основного свойства существует набор свободных порождающих $\left\{f_{\gamma} \mid \gamma \in \Gamma\right\}$ подалгебры $F(\boldsymbol{a})$ такой, что множество $\left\{d_{e} f_{\gamma} \mid \gamma \in \Gamma\right\}$ является базисом пространства $\mathfrak{b}$, так как его элементы лежат в $\mathfrak{b}$ и линейно независимы. Из леммы 3 следует, что множество $\left\{\pi\left(Q\left(f_{\gamma}\right)\right) \mid \gamma \in \Gamma\right\}$ свободно порождает $S(\mathfrak{b})$. Таким образом, $\left.\pi \circ Q\right|_{F(\boldsymbol{a})}-$ изоморфизм. Так как $\pi: S(\mathfrak{q})^{h_{0}} \rightarrow S(\mathfrak{b})$ является изоморфизмом, то и $\left.Q\right|_{F(\boldsymbol{a})}: F(\boldsymbol{a}) \rightarrow S(\mathfrak{q})^{h_{0}}$ - изоморфизм.

6. ДокаЗАтЕЛЬСтво теоремы 2. Ясно, что если $w \in P(\mathfrak{g})$ и $\{w, F(\boldsymbol{a})\}=0$, то $w \in P(\mathfrak{g})^{\mathfrak{h}}$. Пусть $I=\left\{x \in P(\mathfrak{g})^{\mathfrak{h}} \mid Q(x)=0\right\}$. Ясно, что $I$ - идеал алгебры $P(\mathfrak{g})^{\mathfrak{h}}$. В силу леммы 4 имеет место разложение $P(\mathfrak{g})^{\mathfrak{h}}=F(\boldsymbol{a}) \oplus I$. По лемме 1 подалгебра $F(\boldsymbol{a})$ алгебраически замкнута в $P(\mathfrak{g})^{\mathfrak{h}}$. Поскольку алгебра $F(\boldsymbol{a})$ имеет максимально возможную степень трансцендентности среди коммутативных подалгебр алгебры Ли $P(\mathfrak{g})^{\mathfrak{h}}$, то отсюда следует, что $F(\boldsymbol{a})$ является максимальной коммутативной подалгеброй.

\section{СПИСОК ЛИТЕРАТУРЫ}

[1] А. С. Мищенко, А. Т. Фоменко // Изв. АН СССР. Сер. матем. 1978. Т. 42. № 2. С. 396-415. [2] Э. Б. Винберг // Изв. АН СССР. Сер. матем. 1990. Т. 54. №1. С. 3-25. [3] В. В. Шувалов. О пределах подалгебр Мищенко-Фоменко в алгебрах Пуассона полупростых алгебр Ли // Функц. анализ и его прил. 2002. Т. 36. № 4 (в печати).

Московский государственньй

Принято редколлегией университет им. М. В. Ломоносова

21.08 .2002 